\title{
Time-series of Dual-polarimetric Synthetic Aperture Radar Data to Observe Oil Seeps
}

\author{
Carina Regina de Macedo(1), Ferdinando Nunziata(1), Domenico Velotto(2), Maurizio \\ Migliaccio(1), \\ (1) Università degli Studi di Napoli Parthenope - Dipartimentio Ingegneria, Napoli, ITALY \\ Email: \{carinaregina.demacedo, ferdinando.nunziata, maurizio.migliaccio\} \\ @uniparthenope.it \\ (2) German Aerospace Center - IMF-SAR BF, Bremen, GERMANY \\ Email: Domenico.velotto@dlr.de
}

\begin{abstract}
In this study, SAR oil spill observation is investigated for different polarizations, incidence angles and wind conditions. The influence of polarization, wind speed, and incidence angle for sea oil slick detection has been widely studied. Nevertheless, such experiments are frequently undertaken not using a large dataset and with the additional drawback of being collected by different SAR sensors and/or in different locations. The dataset used in this study is composed by a time-series of 42 TerraSAR-X dual-pol SAR images acquired between July 2011 and April 2016 over the same geographical location. The SAR oil spill observation dependency on those parameters is shown for the first time using a large time-series of the same oil type. Experimental results show that the oil seepage observation is impaired with increasing the incidence angle and strongly depends on wind conditions. However, those effects are more pronounced using the single polarization channels, while they are mitigated by using dual polarimetric channels.

The experiments show that the wind speed, polarization and incidence angle impact the oil observation. The global trend shows that the dual-polarization feature works better than the single polarization one.
\end{abstract}

